\title{
Loss of ARID1A Expression is Associated with Systemic Inflammation Markers and has Important Prognostic Significance in Gastric Cancer.
}

\section{Xuan Wang}

Nanjing Medical University

\section{Keying Che}

Nanjing Drum Tower Hospital: Nanjing University Medical School Affiliated Nanjing Drum Tower Hospital

\section{Tao Shi}

Nanjing Drum Tower Hospital: Nanjing University Medical School Affiliated Nanjing Drum Tower Hospital

Qin Liu

Nanjing Drum Tower Hospital: Nanjing University Medical School Affiliated Nanjing Drum Tower Hospital

\section{Xinyun Xu}

Nanjing Drum Tower Hospital: Nanjing University Medical School Affiliated Nanjing Drum Tower Hospital

\section{Hongyan Wu}

Nanjing Drum Tower Hospital: Nanjing University Medical School Affiliated Nanjing Drum Tower Hospital

\section{Lixia Yu}

Nanjing Drum Tower Hospital: Nanjing University Medical School Affiliated Nanjing Drum Tower Hospital

\section{Baorui Liu}

Nanjing Drum Tower Hospital: Nanjing University Medical School Affiliated Nanjing Drum Tower Hospital

\section{Jia Wei ( $\nabla$ jiawei99@nju.edu.cn )}

The Comprehensive Cancer Centre of Nanjing Drum Tower Hospital, The Affiliated Hospital of Nanjing University Medicine School https://orcid.org/0000-0003-3024-8878

\section{Research Article}

Keywords: gastric cancer, ARID1A, systemic inflammation markers, immunotherapy, nomogram 
Posted Date: November 23rd, 2021

DOl: https://doi.org/10.21203/rs.3.rs-1090858/v1

License: (9) This work is licensed under a Creative Commons Attribution 4.0 International License. Read Full License

Version of Record: A version of this preprint was published at Journal of Cancer Research and Clinical Oncology on March 16th, 2022. See the published version at https://doi.org/10.1007/s00432-022-03971W. 


\section{Abstract}

Background: The tumor suppressor gene AT-rich interactive domain 1A (ARID1A) and systemic inflammatory response (SIR) have been reported to be related to the sensitivity of immunotherapy. This study intended to explore the relationship between $A R I D 1 A$ expression and SIR, and to further elucidate the prognostic value of $A R I D 1 A$ expression in gastric cancer (GC).

Methods: The mRNA and protein expression of ARID1A were detected in 272 formalin-fixed paraffinembedded (FFPE) tumor tissues. The data of nine systemic inflammation markers were collected one week before gastrectomy. Univariate and multivariate COX analysis were used to screen out independent predictors of GC.

Results: Negative expression of ARID1A protein was related to GC with microsatellite instability-high (MSI$H)(p=0.033)$, positive programmed cell death-ligand $1(P D-L 1)(p=0.005)$ and lower albumin level $(\mathrm{p}=0.0064)$. Low expression of ARID1A mRNA was common in GC with abnormal E-cadherin $(p=0.020)$ and higher platelet/lymphocyte ratio (PLR) $(p=0.0391)$. Multivariate COX analysis showed that the expression of ARID1A protein $(p=0.023)$, age $(p=0.004)$, T stage $(p=0.009)$ and $N$ stage $(p=0.009)$ were independent predictors of GC. The nomogram established by independent predictors can accurately evaluate the survival risk of patients with GC.

Conclusions: The loss of ARID1A protein expression was associated with MSI-H subtype and high expression of PD-L1 in GC. Negative ARID1A protein and low expression of mRNA were associated with aberrant systemic inflammatory markers. Expression of ARID1A protein had important prognostic significance in GC.

\section{Introduction}

Gastric cancer (GC) is the sixth most common type of malignancy and the fourthleading cause of global cancer-related death(Sung et al. 2021). In recent years, various clinical trials have shown that the immune checkpoint blockade (ICB) therapy using anti-programmed cell death 1 (PD-1)/programmed cell deathligand 1 (PD-L1) antibodies has achieved remarkable treatment outcomes in GC(Fuchs et al. 2018; Janjigian et al. 2021; Y. Kang et al. 2017; Y. K. Kang et al. 2017). However, only a small part of patients can respond to anti-PD-1/PD-L1 antibodies. Biomarkers must be found to selected patients that might benefit from immune checkpoint inhibitors (ICIs). KETNOTE-059 trial showed that pembrolizumab had better therapeutic effect in gastric or EGJ cancer patients with PD-L1 combined positive score (CPS) $\geq 1$ than $\mathrm{CPS}<1$ (Objective response rate (ORR) 15.5\% and 6.4\% respectively)(Fuchs et al. 2018) and pembrolizumab was approved by the Food and Drug Administration (FDA) for the treatment of PD-L1 positive recurrent or gastric or EGJ cancer with in 2017. KETNOTE-062 demonstrated that pembrolizumab can significantly improve overall survival (OS) in patients whose tumors expressed PD-L1 (CPS $\geq 10$ ) compared with chemotherapy (17.4 months vs 10.8 months)(Shitara et al. 2018). CHECKMATE-649 has revealed that nivolumab combined with chemotherapy have more superior OS than chemotherapy alone 
in advanced GC and EGJ cancer(13.8 months vs 11.6 months) especially when PD-L1 CPS $\geq 5$ (14.4 months vs 11.1 months), therefore nivolumab plus chemotherapy were approved by the FDA as a new standard first-line treatment for advanced GC and EGJ cancer in 2021(Janjigian et al. 2021). These remarkable results illustrated the value of PD-L1 expression level in guiding the use of ICls. Another research showed that patients with microsatellite instability-high (MSI-H) or Epstein-Barr virus (EBV)positive metastatic GCs had a significantly higher overall response rate (ORR) to pembrolizumab (85.7\% and $100 \%$, respectively)(S. T. Kim et al. 2018). In KEYNOTE-061 and KEYNOTE-062, the estimated 12month OS rates of patients with MSI-H GCs receiving pembrolizumab monotherapy were significantly higher than that of MSI-H patients receiving chemotherapy (73\% vs 25\% in KEYNOTE-061 and $79 \%$ vs 47\% in KEYNOTE-062)(Chao et al. 2021). In a recent phase lb/II clinical trial (NCT02915432), high tumor mutational burden (TMB-H) was significantly associated with better OS compared low tumor mutational burden (TMB-L) group in advanced GC receiving immunotherapy (14.6 months vs 4.0 months)(Wang et al. 2019). These studies provided reliable basis for identifying biomarkers of ICB therapy. Systemic inflammatory response (SIR) was reported to have prognostic value for patients receiving ICls(Formica et al. 2020; Jiang et al. 2018). A recent meta-analysis concluded that elevated blood neutrophil/lymphocyte ratio (NLR) before treatment was a promising prognostic biomarker for advanced cancer patients receiving immunotherapy(Jiang et al. 2018). Gastric Inflammatory Prognostic Index (GIPI) combining NLR, CRP, and albumin established by Formica et al had significantly prognostic value in metastatic EGJ/GC patients receiving ICIs(Formica et al. 2020).

AT-rich interactive domain $1 \mathrm{~A}(A R I D 1 A)$ is an important subunit of the Switch/Sucrose Non-fermentable (SWI/SNF) chromatin remodeling complex. ARID1A is frequently mutated among a wide variety of tumors, such as ovarian clear cell carcinoma (40\%-57\%) (S. Jones et al. 2010; Wiegand et al. 2010), GC (18.7\%-23\%)(Cho et al. 2019; S Jones et al. 2012), hepatocellular carcinoma (10\%-17\%)(Fujimoto et al. 2012; Guichard et al. 2012) and breast cancer (5\%-15\%) (Liang et al. 2018; Mamo et al. 2012), and its mutations usually lead to loss of expression(Wu et al. 2014). ARID1A mutations are frequently present in EBV positive GC("Comprehensive molecular characterization of gastric adenocarcinoma" 2014), and ARID1A negative expression was tightly correlated with PD-L1 positive and can increase the expression of PD-L1 via activating PI3K/AKT signaling pathway(Y. B. Kim et al. 2019). ARID1A deficiency has been reported to cause mismatch repair (MMR) protein dysfunction and was associated with MSI subtype and TMB-H(Shen et al. 2018). Though loss of ARID1A was considered a marker of poor prognosis(Yang et al. 2016), ARID1A alteration was an independent predictor of longer progression-free survival (PFS) after ICB therapy in many kinds of cancers(Okamura et al. 2020). Tumor infiltrating lymphocytes (TILs) is also an important factor affecting the efficacy of immunotherapy, and loss of ARID1A protein in both tumors and paired-adjacent normal peritumor mucosa tissues was correlated with increased TILs(Zou et al. 2020).

In this study, we aimed to figure out the relationship between the expression pattern of $A R I D 1 A$ and clinicopathological characteristics and other markers associated with the efficacy of ICB in gastric cancer patients. Furthermore, we evaluated the prognostic significance of ARID1A expression pattern in GC patients, and established a nomogram based on ARID1A protein expression and clinical variables to predict the survival risk of patients with GC. 


\section{Methods}

\section{Patients}

We retrieved clinicopathological characteristics from patients with gastric adenocarcinoma who underwent D2 gastrectomy between August 2017 and January 2019 in Nanjing Drum Tower Hospital. Patient characteristics of age, gender, TNM stage (according to the 8th edition of the American Joint Committee on Cancer (AJCC)), lymph node metastasis, vascular invasion and neural invasion were included in the study. Nine systemic inflammatory markers including platelet, monocyte, and lymphocyte count; neutrophil/lymphocyte ratio (NLR), platelet/lymphocyte ratio (PLR), lymphocyte/ monocyte ratio (LMR); and albumin, lactate dehydrogenase (LDH) and C-reactive protein (CRP) within 1 week prior to gastrectomy were collected.

\section{Immunohistochemical (IHC) staining of tumor tissues}

IHC staining of ARID1A, MutL homolog 1(MLH1), postmeiotic segregation increased 2 (PMS2), PD-L1, vascular endothelial growth factor receptor 2(VEGFR2), p53, E-cadherin and alpha-fetoprotein (AFP) was performed with formalin-fixed paraffin-embedded (FFPE) tissue sections ( $2 \mu \mathrm{m}$ thick) from the representative tumor tissue block. Briefly, the sections were deparaffinized in xylene and rehydrated with gradient ethanol $(100 \%, 95 \%$, and $80 \%)$, and washed with PBS. Then, $0.3 \%$ hydrogen peroxide in methanol was used for 30 minutes to block activity of endogenous peroxidase. After washing with PBS, the sections were incubated with primary antibodies at $4^{\circ} \mathrm{C}$ overnight. The following primary antibodies were used: anti-ARID1A (\#12354; Cell Signaling Technology, USA), anti-PD-L1 (SP142; Spring Bio, USA), antiMLH1 (ES05; Dako, USA), anti-PMS2 (EP51; Dako, USA), anti p53 (D07; Zhongshan Golden Bridge Biotechnology(ZSGB-BIO), China), anti E-cadherin (EP6; ZSGB-BIO), anti AFP (EP209; ZSGB-BIO), anti VEGFR2 (rabbit monoclonal; ZSGB-BIO). After then, the sections were incubated with secondary antibodies for $30 \mathrm{~min}$ at room temperature. The staining was visualized with diaminobenzidine and counterstained with hematoxylin.

The IHC score of ARID1A was calculated on the basis of the percentage of positive nuclear staining and staining intensity (Zhu et al. 2018). The percentage of stained cells in the sections was divided into five grades: $0,1,2,3$, and 4 for $\leq 5 \%, 6 \%-30 \%, 31 \%-50 \%, 51 \%-75 \%$, and $>75 \%$ stained cells, respectively. Staining intensity was divided into four grades: $0,1,2$, and 3 for no staining, weak staining, moderate staining, and strong staining, respectively. The total IHC score (0-12) =percentage of positive nuclear staining $\times$ staining intensity. The expression level of ARID1A was defined as negative group (score $\leq 1)$ and positive group (score $>1$ ). The evaluation of PD-L1 was based on CPS, and CPS $\geq 1$ was defined as PD-L1 positive. Tumors showing complete loss of nuclear MHL1 or PMS2 were classified as MHL1 or PMS2 aberrant(Setia et al. 2016). Aberrant p53 expression was defined as complete loss or diffuse and strong p53 nuclear positivity(Ahn et al. 2017; Setia et al. 2016). Aberrant E-cadherin expression was defined as complete loss of membranous expression or markedly reduced membranous staining $(>30 \%)$ (Ahn et al. 2017).. AFP was considered positive when $\geq 1 \%$ tumor cells was stained(Kinjo et al. 2012). VEGFR2 staining levels were defined as follows: $-(<5 \%$ positive cells $),+(\geq 5 \%$ and $<50 \%$ positive cells $),+$ 
$+(\geq 50 \%$ and $<75 \%$ positive cells $)$, and $+++(\geq 75 \%$ positive cells $)$. The staining results were evaluated independently by two experienced pathologists.

\section{EBV-encoded small RNA in-situ hybridization}

EBV-encoded RNA (EBER) was detected using automated EBER staining method with Ventana Benchmark in situ hybridization system (Ventana Medical Systems) according to the manufacturer's protocol. When $>20 \%$ of the tumor cells showed EBER stained, the case was defined as EBER positive.

\section{mRNA extraction and quantitative real-time polymerase chain reaction (qRT-PCR).}

Total RNA was extracted from FFPE tumor tissues according to a proprietary procedure (European patent number EP1945764-B1). After extraction and purification, the total RNA was used to generate cDNA with M-MLV Reverse Transcriptase Kit (Invitrogen, Carlsbad, CA, USA). The expression of ARID1A and $\beta$-actin (used as endogenous control) was detected by qRT-PCR using QuantStudio ${ }^{\mathrm{TM}} 7$ Flex (Applied Biosystems) following conditions: one cycle at $95^{\circ} \mathrm{C}$ for $20 \mathrm{~s}, 40$ cycles of $95^{\circ} \mathrm{C}$ for $1 \mathrm{~s}$ and $60^{\circ} \mathrm{C}$ for $20 \mathrm{~s}$. Taqman $^{\text {TM }}$ Fast Advanced Master Mix (Thermo Fisher Scientific, \#4444556) and probes of ARID1A (Thermo Fisher Scientific, Hs00195664_m1) and ß-actin (Thermo Fisher Scientific, Hs03023943_g1) were used for amplification of the cDNA.

\section{Classification of molecular subtypes}

We divided GC into five molecular subtypes according to previous research(Setia et al. 2016): EBV positive GC, MSI-H GC, GC with aberrant expression of E-cadherin, GC with aberrant expression of p53 and GC with p53 expression in normal. EBV status was determined via in situ hybridization as described above. Cases with aberrant MLH1 or PMS2 were defined as MSI-H. The remaining cases were divided into E-cadherin aberrant expression subtype, p53 aberrant expression subtype and p53 normal expression subtype according to the expression of E-cadherin and p53.

\section{Statistical analysis.}

ARID1A protein expression and clinical variables including gender, age, stage, $\mathrm{T}$ stage, $\mathrm{N}$ stage and tumor size were involved in univariate Cox analysis to select OS related variables. Significant variables in the univariate Cox analysis were further incorporated into the multivariate Cox analysis to determine independent prognostic predictors. The nomograms were established with independent prognostic predictors selected by multivariate Cox analysis to predict individual probabilities of clinical events. The Harrell's concordance index(C-index) was used to quantify the discrimination of the nomogram and the calibration curve was used to measures the discrepancy between the predicted probabilities and the actual survival(Hanley \& McNeil 1983). All statistical analysis were carried out using IBM SPSS version 25.0 (IBM, SPSS Statistics) and R version 3.6.2. 


\section{Results}

\section{Expression of ARID1A protein was associated with mRNA level}

All the patients were divided into ARID1A negative group $(n=58,21.3 \%)$ and ARID1A positive group ( $\mathrm{n}=214,78.7 \%$ ) by IHC (Fig.1a, b). The mRNA expression of ARID1A was detected in 236 samples by qRTPCR and relative expression level of mRNA was calculated according to the formula $2^{-\Delta \Delta C t}$. T test analysis showed that mRNA expression level of ARID1A protein negative group was significantly lower than that of protein positive group (Fig.1c).

\section{Different protein expression levels of ARID1A in the five GC molecular subtypes}

Among the 272 GC patients, 3 cases (1.1\%) were EBV positive, 9 cases (3.3\%) were MSI-H subtype, 33 cases $(12.1 \%)$ were E-cadherin aberrant, 164 cases $(60.3 \%)$ were p53 aberrant and 63 cases $(23.2 \%)$ were p53 normal (Figure. 2a). The probability of ARID1A protein negative in MSI-H subtype (5/9, 55.6\%) was significantly higher than that in p53 aberrant subtype $(33 / 164,20.1 \%, p=0.037)$ and p53 normal subtype $(12 / 63,19.0 \%, p=0.046$, Figure. $2 b)$. Among the 3 patients with EBV positive GC, 1 patient was negative for ARID1A protein. There was no difference in the proportion of negative ARID1A protein between EBV positive GC and other subtypes due to the small sample size.

\section{ARID1A expression pattern and clinicopathological characteristics of patients}

Clinicopathological characteristics of $272 \mathrm{GC}$ patients were summarized in the Table 1 . Until now, no study has yet clearly established the grading standard for the relative expression level of ARID1A mRNA extracted from FFPE, and the loss of expression caused by ARID1A mutation has special value for research. Considering that the mutation rate of $A R I D 1 A$ in GC is $18.7 \%-23 \%$, in this study, we arranged the relative expression level of $A R I D 1 A$ mRNA in ascending order, and the first $20 \%$ cases were defined as low expression of ARID1A mRNA ( $\mathrm{n}=47,20 \%$ ), and the remaining $80 \%$ cases were defined as high expression of ARID1A mRNA ( $\mathrm{n}=193,80 \%$ ). The protein or mRNA expression of ARID1A was not associated with gender, age, Body Mass Index (BMI), stage, depth of invasion, lymph node metastasis, distant metastasis, perineural invasion or vascular invasion. However, loss expression of ARID1A protein was significantly correlated with MSI-H subtype, PD-L1 CPS $\geq 1$ and CPS $\geq 10$ ( $p=0.033, p=0.005$ and $p=0.012$, respectively). In addition, among 47 patients with low expression of ARID1A mRNA, there were 20 (42.6\%) patients with PD-L1 CPS $\geq 10$ and only $29.6 \%$ (56/189) patients with high expression of ARID1A mRNA were PD-L1 $\mathrm{CPS} \geq 10$, although the difference was not statistically significant. The low expression of ARID1A mRNA 
was correlated with abnormal E-cadherin $(p=0.003)$. There was no correlation between the expression of p53, AFP or VEFGR2 and the expression of ARID1A protein or mRNA.

\section{Relationship between ARID1A expression pattern and systemic inflammatory markers of patients}

Both SIR and ARID1A were reported to have predictive value in the prognosis of immunotherapy(Formica et al. 2020; Okamura et al. 2020), so we investigated whether there is any association between ARID1A expression and systemic inflammatory markers. ARID1A negative protein group had a lower level of albumin ( $p=0.0064$, Figure. 3e), while the low mRNA group had a higher level of PLR $(p=0.0391$, Figure. $3 i)$. In addition, the LMR level appeared to be lower in the ARID1A negative protein group, but it was not statistically significant ( $p=0.0689$, Figure. $3 b$ ). We also analyzed the relationship between systemic inflammatory markers and ARID1A expression by the chi-square test (Table S1), and found ARID1A protein negative was associated with high level of PLR and low level of albumin $(p=0.038$ and $p=0.008$, respectively, Table S1). Moreover, ARID1A mRNA was low in 17 (17.7\%) of the 96 cases with low LMR, and in 30 (32.3\%) of the 93 patients with high LMR, but the difference was not statistically significant $(p=0.073$, Table S1).

\section{ARID1A expression pattern was significantly associated with overall survival}

Follow-up of 172 patients was carried out up to the date of death from any cause or last contact, and the median survival time was 23 months. Both negative expression of ARID1A protein and low expression of ARID1A mRNA were significantly associated with poor prognosis ( $\mathrm{p}=0.002$ and $\mathrm{p}=0.013$, respectively) (Figure $4 a, b)$. Univariate and multivariate Cox proportional hazard models were performed to assess the value of ARID1A protein expression pattern and clinical variables on survival (Table 2). Univariate analysis demonstrated that negative expression of ARID1A protein $(p=0.002)$, older age $(p=0.003)$, higher T stage $(p=0.002)$, and higher $N$ stage $(p=0.003)$ were associated with decreased OS. Multivariate Cox analysis indicated that negative expression of ARID1A protein $(p=0.023)$, older age $(p=0.004)$, higher $T$ stage $(p=0.009)$, or higher $N$ stage $(p=0.009)$ were independent risk factors for poor prognosis.

\section{Development and validation of the nomogram}

To predict the survival risk of patients with GC, a nomogram was established based on all independent risk factors selected by multivariate Cox analysis (Figure. 5a). The C-index of the nomogram was 0.719 (95\% Cl: 0.683-0.755), which was higher than $8^{\text {th }}$ edition of the AJCC staging system (C-index $=0.657,95 \%$ Cl: 0.621-0.692). In addition, the calibration plots of our nomogram showed high consistency between the nomogram-predicted outcomes and the observational outcomes (Figure. 5b, c). 


\section{Discussion}

In previous researches, the mutation rate of tumor suppressor gene ARID1A was $18.7 \%-23 \%$ in GC (Cho et al. 2019; S Jones et al. 2012), and its mutations often led to loss of protein expression, which made ARID1A a poor therapeutic target. In this study, we evaluated the protein and mRNA expression of ARID1A by IHC staining and qRT-PCR respectively. 58 (21.3\%) among 272 patients were assessed as negative ARID1A protein. We found that the expression level of ARID1A mRNA was also lower in protein negative group.

GC is a malignant tumor with complex pathogenic factors and high heterogeneity. The Cancer Genome Atlas (TCGA) divided GC into four molecular subtypes: EBV positive subtype, MSI subtype, chromosomal instability (CIN) subtype and genomically stable (GS) subtype("Comprehensive molecular characterization of gastric adenocarcinoma" 2014). This study found that EBV positive GC had higher frequency of ARID1A mutation (55\%), extreme DNA hypermethylation and amplification of JAK2 and PDL1. MSI-H GC showed elevated mutation rates and MLH1 gene silencing. GS subtype was mostly histological diffuse type with high frequency of $\mathrm{CDH} 1$ and RHOA mutations. CIN subtype showed obvious aneuploidy and was mostly intestinal type or gastroesophageal borderline tumor("Comprehensive molecular characterization of gastric adenocarcinoma" 2014). In 2016, Setia et al proposed a more convenient classification of GC subtypes using IHC and ISH: EBV positive subtype, MSI-H subtype, Ecadherin abnormal subtype, p53 abnormal subtype and p53 normal subtype GC(Setia et al. 2016). It was also found that EBV positive GC had higher PD-L1 expression and GC with abnormal E-cadherin expression was associated with histological diffuse type. GC with abnormal p53 expression was associated with histological intestinal type. EBV positive and MSI-H type GC had better prognosis. In our study, 272 cases with GC were divided into the above-mentioned 5 subtypes according to the detection of EBER and IHC of MLH1, PMS2, E-cadherin and p53. We found that the negative rates of ARID1A protein in the MSI-H subtype $(5 / 9,55.6 \%)$ was significantly higher than that in the p53 abnormal subtype $(33 / 164$, $20.1 \%, p=0.037)$ and the $p 53$ normal subtype $(12 / 63,19.0 \%, p=0.046)$. Among the three EBV positive cases, one case was negative for ARID1A protein. Due to the small sample size of EBV positive cases, we couldn't find the difference of ARID1A protein negative rates between EBV positive GC and other subtypes.

According to previous studies(Y. B. Kim et al. 2019), loss of ARID1A protein expression was significantly associated with higher PD-L1 expression in GC, which was consistent with our findings. Considering that GC with MSI-H and positive PD-L1 expression had better response to ICls and negative expression of ARID1A protein was significantly related to MSI-H and PD-L1 positive(Fuchs et al. 2018; S. T. Kim et al. 2018; Y. B. Kim et al. 2019; Shen et al. 2018), expression of ARID1A may become a biomarker for immunotherapy. Reduced expression of ARID1A was reported to down-regulate the transcription of $\mathrm{CDH} 1$ and enhance tumor invasion(Yan et al. 2014), and our study found lower ARID1A mRNA level was related to aberrant E-cadherin expression. 
Cancer-related inflammation is a key determinant of tumor progression and metastasis in most cancers(Hanahan \& Weinberg 2011). With the rapid development of immunotherapy, some systemic inflammatory markers (such as NLR, PLR, CRP, albumin, etc) have been found to be closely related to the prognosis of ICls in different kinds of tumors(Dharmapuri et al. 2020; Formica et al. 2020; Rossi et al. 2020). ARID1A mutation has been reported as an independent predictor of longer PFS during tumor immunotherapy(Okamura et al. 2020), and is closely related to increased expression of gene signatures of immune checkpoint, cytotoxic T-cell function and antigen presentation(Mehrvarz Sarshekeh et al. 2021). Increased expression of key genes (HAVCR2, IDO1, IL4I1, LAG3, PDCD1, PDCD1LG and THFRSF4) which are known to be related to immune response are also associated with ARID1A mutation(Mehrvarz Sarshekeh et al. 2021). In our study, we found loss of ARID1A protein was associated with lower albumin $(\mathrm{p}=0.0064)$ and low ARID1A mRNA was associated with higher PLR $(\mathrm{p}=0.0689)$. Since both ARID1A and SIR have prognostic value in tumor immunotherapy, the mechanism of their interrelation deserves further study to provide more information for the research of ICls treatment biomarkers.

In clinical practice, the TNM staging system is usually used to assess the prognosis of patients with malignant tumors. However, the current staging system is not sufficient to predict the prognosis. It is essential to establish more accurate clinical prognostic tools for GC. In our research, we established a nomogram based on four independent predictors(including expression of ARID1A protein, age, T stage and $\mathrm{N}$ stage) for OS as a practical prognostic tool. Nomogram quantifies the risk by including all clinical and pathological variables related to the prognosis, which can be used for individualized prognosis prediction(Graesslin et al. 2010; Han et al. 2012). Our research was the first to combine ARID1A and clinicopathological characteristics to establish a nomogram for survival prediction in GC. Furthermore, we compared the predictive accuracy between our nomogram and the 8th edition of AJCC TNM staging system, and found our nomogram was superior to the TNM staging system in predicting the prognosis of OS in GC patients. These results showed that our nomogram was relatively good at identifying high-risk populations and predicting prognosis.

There were some limitations in our study. Firstly, in 36 of 272 samples, we could not detect the expression of the target genes (including ARID1A and $\beta$-actin) by qRT-PCR using total RNA extracted from FFPE samples due to the degradation of RNA. Secondly, our samples lacked the detection data of GS and CIN, so the association between ARID1A expression and TCGA subtypes couldn't be analyzed. Thirdly, we were unable to obtain information on postoperative adjuvant treatments, such as chemotherapy, radiotherapy, immunotherapy or targeted therapy, so we couldn't compare the relationship between ARID1A expression and prognosis in different kinds of treatments.

\section{Conclusion}

In conclusion, we found that ARID1A protein negative group had lower level of mRNA and was significantly associated with PD-L1 positive and aberrant systemic inflammatory markers. The established nomogram based on ARID1A, age, T stage and N stage was a practical tool for evaluating the prognosis of GC. 


\section{Declarations}

\section{Funding}

This work was funded by grants from National Natural Science Foundation of China (82073382) and the Distinguished Young Scholars of Jiangsu Province (BK20190001). The funding sources had no role in the study design, data collection, data analysis, data interpretation, or writing of this review. The funding sources have no involvement in study design; the collection, analysis and interpretation of data; in the writing of the report; or in the decision to submit the paper for publication.

\section{Competing interests}

The authors have no conflicts of interest to declare.

\section{Authors' contributions}

Xuan Wang: Drafting the article; Keying Che: Revising it critically for important intellectual content; Tao Shi: The acquisition of data; Qin Liu: Visualization; Xinyun Xu: Analysis and interpretation of data; Hongyan Wu: Analysis and interpretation of data; Lixia Yu: The acquisition of data; Baorui Liu: Supervision, final approval of the version to be submitted; Jia Wei: Conceptualization, funding acquisition.

\section{Data Availability}

The data described in this manuscript are contained in published articles or available from the corresponding author upon reasonable request.

\section{Ethics approval}

This study was conducted in accordance with the code of ethics of the World Medical Association (Declaration of Helsinki) and approved by the Ethics Committee of Nanjing Drum Tower Hospital (No. 2016-196-01).

\section{Consent to participate}

Informed consent was obtained from all individual participants included in the study.

\section{Consent to publish}




\section{Acknowledgements}

The authors would like to thank all patients who participated in this study.

\section{References}

1. Ahn, S, Lee, S, Kim, Y, et al. (2017). High-throughput Protein and mRNA Expression-based Classification of Gastric Cancers Can Identify Clinically Distinct Subtypes, Concordant With Recent Molecular Classifications. The American journal of surgical pathology. https://doi.org/10.1097/pas.0000000000000756

2. Chao, J, Fuchs, C, Shitara, K, et al. (2021). Assessment of Pembrolizumab Therapy for the Treatment of Microsatellite Instability-High Gastric or Gastroesophageal Junction Cancer Among Patients in the KEYNOTE-059, KEYNOTE-061, and KEYNOTE-062 Clinical Trials. JAMA oncology. https://doi.org/10.1001/jamaoncol.2021.0275

3. Cho, J, Ahn, S, Son, DS, et al. (2019). Bridging Genomics and Phenomics of Gastric Carcinoma. Int J Cancer. https://doi.org/10.1002/ijc.32228

4. Comprehensive molecular characterization of gastric adenocarcinoma. (2014). Nature. https://doi.org/10.1038/nature13480

5. Dharmapuri, S, Özbek, U, Lin, J, et al. (2020). Predictive value of neutrophil to lymphocyte ratio and platelet to lymphocyte ratio in advanced hepatocellular carcinoma patients treated with anti-PD-1 therapy. Cancer medicine. https://doi.org/10.1002/cam4.3135

6. Formica, V, Morelli, C, Patrikidou, A, et al. (2020). Gastric Inflammatory Prognostic Index (GIPI) in Patients with Metastatic Gastro-Esophageal Junction/Gastric Cancer Treated with PD-1/PD-L1 Immune Checkpoint Inhibitors. Targeted oncology. https://doi.org/10.1007/s11523-020-00723-z

7. Fuchs, CS, Doi, T, Jang, RW, et al. (2018). Safety and Efficacy of Pembrolizumab Monotherapy in Patients With Previously Treated Advanced Gastric and Gastroesophageal Junction Cancer: Phase 2 Clinical KEYNOTE-059 Trial. JAMA Oncol. https://doi.org/10.1001/jamaoncol.2018.0013

8. Fujimoto, A, Totoki, Y, Abe, T, et al. (2012). Whole-genome sequencing of liver cancers identifies etiological influences on mutation patterns and recurrent mutations in chromatin regulators. $\mathrm{Nat}$ Genet. https://doi.org/10.1038/ng.2291

9. Graesslin, O, Abdulkarim, B, Coutant, C, et al. (2010). Nomogram to predict subsequent brain metastasis in patients with metastatic breast cancer. Journal of clinical oncology : official journal of the American Society of Clinical Oncology. https://doi.org/10.1200/jco.2009.24.6314

10. Guichard, C, Amaddeo, G, Imbeaud, S, et al. (2012). Integrated analysis of somatic mutations and focal copy-number changes identifies key genes and pathways in hepatocellular carcinoma. Nat Genet. https://doi.org/10.1038/ng.2256 
11. Han, D, Suh, Y, Kong, S, et al. (2012). Nomogram predicting long-term survival after d2 gastrectomy for gastric cancer. Journal of clinical oncology : official journal of the American Society of Clinical Oncology. https://doi.org/10.1200/jco.2012.41.8343

12. Hanahan, D, \& Weinberg, R. (2011). Hallmarks of cancer: the next generation. Cell. https://doi.org/10.1016/j.cell.2011.02.013

13. Hanley, J, \& McNeil, B. (1983). A method of comparing the areas under receiver operating characteristic curves derived from the same cases. Radiology. https://doi.org/10.1148/radiology.148.3.6878708

14. Janjigian, Y, Shitara, K, Moehler, M, et al. (2021). First-line nivolumab plus chemotherapy versus chemotherapy alone for advanced gastric, gastro-oesophageal junction, and oesophageal adenocarcinoma (CheckMate 649): a randomised, open-label, phase 3 trial. Lancet (London, England). https://doi.org/10.1016/s0140-6736(21)00797-2

15. Jiang, T, Qiao, M, Zhao, C, et al. (2018). Pretreatment neutrophil-to-lymphocyte ratio is associated with outcome of advanced-stage cancer patients treated with immunotherapy: a meta-analysis. Cancer immunology, immunotherapy : Cll. https://doi.org/10.1007/s00262-018-2126-z

16. Jones, S, Li, M, Parsons, D, et al. (2012). Somatic mutations in the chromatin remodeling gene ARID1A occur in several tumor types. Human mutation. https://doi.org/10.1002/humu.21633

17. Jones, S, Wang, TL, Shih le, M, et al. (2010). Frequent mutations of chromatin remodeling gene ARID1A in ovarian clear cell carcinoma. Science. https://doi.org/10.1126/science.1196333

18. Kang, Y, Boku, N, Satoh, T, et al. (2017). Nivolumab in patients with advanced gastric or gastrooesophageal junction cancer refractory to, or intolerant of, at least two previous chemotherapy regimens (ONO-4538-12, ATTRACTION-2): a randomised, double-blind, placebo-controlled, phase 3 trial. Lancet (London, England). https://doi.org/10.1016/s0140-6736(17)31827-5

19. Kang, YK, Boku, N, Satoh, T, et al. (2017). Nivolumab in patients with advanced gastric or gastrooesophageal junction cancer refractory to, or intolerant of, at least two previous chemotherapy regimens (ONO-4538-12, ATTRACTION-2): a randomised, double-blind, placebo-controlled, phase 3 trial. Lancet. https://doi.org/10.1016/S0140-6736(17)31827-5

20. Kim, ST, Cristescu, R, Bass, AJ, et al. (2018). Comprehensive molecular characterization of clinical responses to PD-1 inhibition in metastatic gastric cancer. Nat Med. https://doi.org/10.1038/s41591018-0101-z

21. Kim, YB, Ahn, JM, Bae, WJ, et al. (2019). Functional loss of ARID1A is tightly associated with high PD-L1 expression in gastric cancer. Int J Cancer. https://doi.org/10.1002/ijc.32140

22. Kinjo, T, Taniguchi, H, Kushima, R, et al. (2012). Histologic and immunohistochemical analyses of afetoprotein-producing cancer of the stomach. The American journal of surgical pathology. https://doi.org/10.1097/PAS.0b013e31823aafec

23. Liang, $X$, Vacher, $S$, Boulai, A, et al. (2018). Targeted next-generation sequencing identifies clinically relevant somatic mutations in a large cohort of inflammatory breast cancer. Breast Cancer Res. https://doi.org/10.1186/s13058-018-1007-x 
24. Mamo, A, Cavallone, L, Tuzmen, S, et al. (2012). An integrated genomic approach identifies ARID1A as a candidate tumor-suppressor gene in breast cancer.

Oncogene. https://doi.org/10.1038/onc.2011.386

25. Mehrvarz Sarshekeh, A, Alshenaifi, J, Roszik, J, et al. (2021). ARID1A Mutation May Define an Immunologically Active Subgroup in Patients with Microsatellite Stable Colorectal Cancer. Clinical cancer research : an official journal of the American Association for Cancer Research. https://doi.org/10.1158/1078-0432.Ccr-20-2404

26. Okamura, R, Kato, S, Lee, S, et al. (2020). ARID1A alterations function as a biomarker for longer progression-free survival after anti-PD-1/PD-L1 immunotherapy. $J$ Immunother Cancer. https://doi.org/10.1136/jitc-2019-000438

27. Rossi, S, Toschi, L, Finocchiaro, G, et al. (2020). Neutrophil and lymphocyte blood count as potential predictive indicators of nivolumab efficacy in metastatic non-small-cell lung cancer. Immunotherapy. https://doi.org/10.2217/imt-2019-0154

28. Setia, N, Agoston, A, Han, H, et al. (2016). A protein and mRNA expression-based classification of gastric cancer. Modern pathology : an official journal of the United States and Canadian Academy of Pathology, Inc. https://doi.org/10.1038/modpathol.2016.55

29. Shen, J, Ju, Z, Zhao, W, et al. (2018). ARID1A deficiency promotes mutability and potentiates therapeutic antitumor immunity unleashed by immune checkpoint blockade. Nat Med. https://doi.org/10.1038/s41591-018-0012-z

30. Shitara, K, Ozguroglu, M, Bang, YJ, et al. (2018). Pembrolizumab versus paclitaxel for previously treated, advanced gastric or gastro-oesophageal junction cancer (KEYNOTE-061): a randomised, open-label, controlled, phase 3 trial. Lancet. https://doi.org/10.1016/S0140-6736(18)31257-1

31. Sung, H, Ferlay, J, Siegel, R, et al. (2021). Global Cancer Statistics 2020: GLOBOCAN Estimates of Incidence and Mortality Worldwide for 36 Cancers in 185 Countries. CA: a cancer journal for clinicians. https://doi.org/10.3322/caac. 21660

32. Wang, F, Wei, XL, Wang, FH, et al. (2019). Safety, efficacy and tumor mutational burden as a biomarker of overall survival benefit in chemo-refractory gastric cancer treated with toripalimab, a PD-1 antibody in phase lb/Il clinical trial NCT02915432. Ann

Oncol. https://doi.org/10.1093/annonc/mdz197

33. Wiegand, K, Shah, S, Al-Agha, O, et al. (2010). ARID1A mutations in endometriosis-associated ovarian carcinomas. The New England journal of medicine. https://doi.org/10.1056/NEJMoa1008433

34. Wu, RC, Wang, TL, \& Shih le, M. (2014). The emerging roles of ARID1A in tumor suppression. Cancer Biol Ther. https://doi.org/10.4161/cbt.28411

35. Yan, H, Wang, X, Zhang, Q, et al. (2014). Reduced expression of the chromatin remodeling gene ARID1A enhances gastric cancer cell migration and invasion via downregulation of E-cadherin transcription. Carcinogenesis. https://doi.org/10.1093/carcin/bgt398

36. Yang, L, Wei, S, Zhao, R, et al. (2016). Loss of ARID1A expression predicts poor survival prognosis in gastric cancer: a systematic meta-analysis from 14 studies. Sci 
Rep. https://doi.org/10.1038/srep28919

37. Zhu, Y, Sheng, L, Wu, J, et al. (2018). Loss of ARID1A expression is associated with poor prognosis in patients with gastric cancer. Human pathology. https://doi.org/10.1016/j.humpath.2018.04.003

38. Zou, J, Qin, W, Yang, L, et al. (2020). Genetic alterations and expression characteristics of ARID1A impact tumor immune contexture and survival in early-onset gastric cancer. American journal of cancer research.

\section{Tables}

\section{Table 1}

Clinicopathological characteristics of GC patients according to ARID1A expression. 


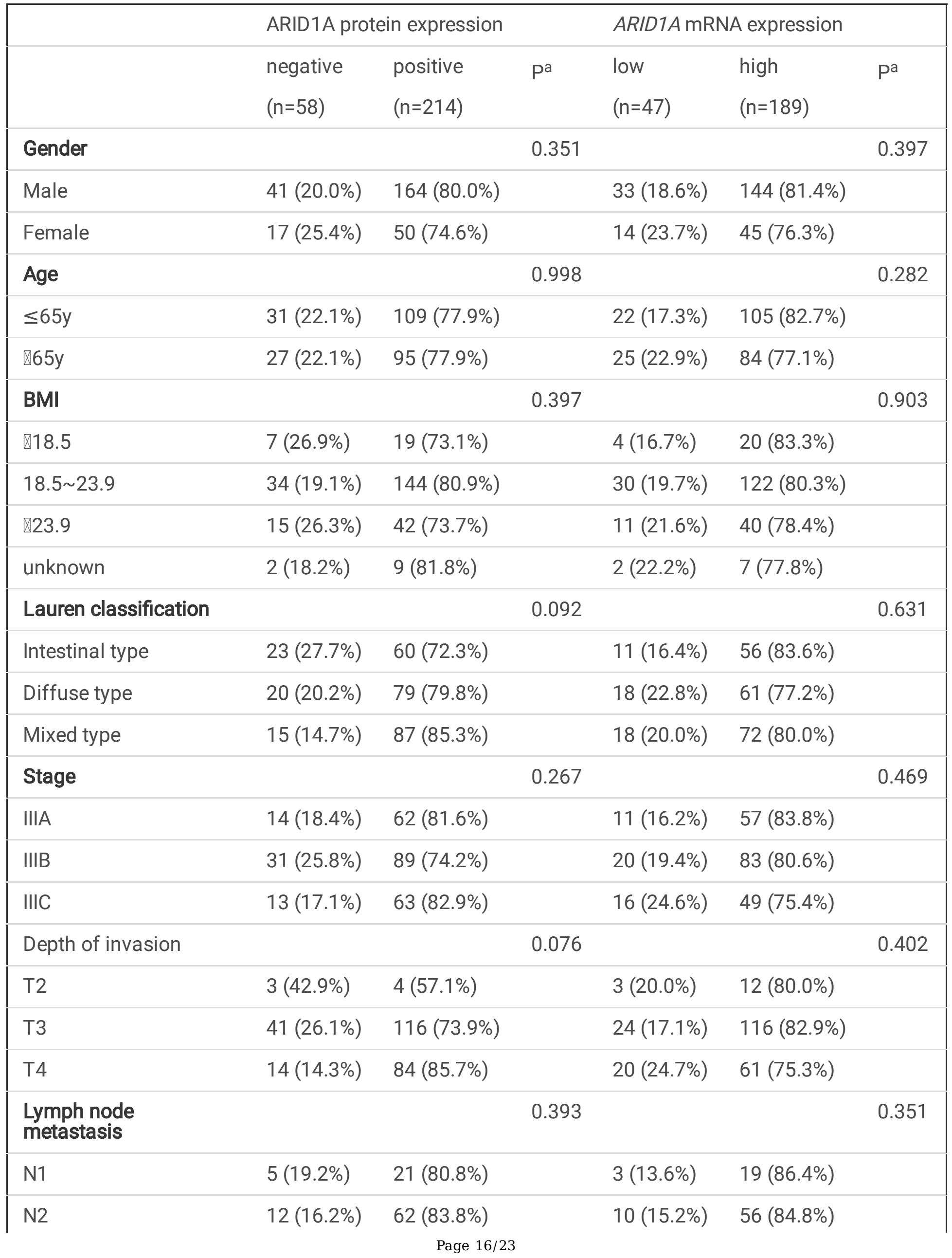




\begin{tabular}{|c|c|c|c|c|c|c|}
\hline N3 & $41(23.8 \%)$ & $131(76.2 \%)$ & & $34(23.0 \%)$ & 114 (77.0\%) & \\
\hline Venous invasion & & & 0.955 & & & 0.435 \\
\hline Present & $46(21.4 \%)$ & $169(78.6 \%)$ & & 39 (21.0\%) & 147 (79.0\%) & \\
\hline Absent & $12(21.1 \%)$ & $45(78.9 \%)$ & & $8(16.0 \%)$ & $42(84.0 \%)$ & \\
\hline Nerve invasion & & & 0.127 & & & 1 \\
\hline Present & $50(20.0 \%)$ & $200(80.0 \%)$ & & 43 (19.9\%) & 173 (80.1\%) & \\
\hline Absent & $8(36.4 \%)$ & $14(63.6 \%)$ & & $4(20.0 \%)$ & $16(80.0 \%)$ & \\
\hline MSI status & & & 0.033 & & & 0.803 \\
\hline MSS & $53(20.2 \%)$ & $210(79.8 \%)$ & & $46(20.3 \%)$ & 181 (79.7\%) & \\
\hline MSI-H & $5(55.6 \%)$ & $4(44.4 \%)$ & & $1(12.5 \%)$ & $8(87.5 \%)$ & \\
\hline \multicolumn{7}{|l|}{ PD-L1 } \\
\hline $\mathrm{CPS} \geq 1$ & $50(25.8 \%)$ & $144(74.2 \%)$ & 0.005 & $34(20.1 \%)$ & 135 (79.9\%) & 0.901 \\
\hline CPS®1 & $8(10.3 \%)$ & 70 (89.7\%) & & $13(19.4 \%)$ & $54(80.6 \%)$ & \\
\hline $\mathrm{CPS} \geq 10$ & $26(30.6 \%)$ & 59 (69.4\%) & 0.012 & $20(26.3 \%)$ & $56(73.7 \%)$ & 0.090 \\
\hline CPS $₫ 10$ & $32(17.1 \%)$ & 155 (82.9\%) & & 27 (16.9\%) & 133 (83.1\%) & \\
\hline p53 & & & 0.930 & & & 0.500 \\
\hline Normal & $17(21.0 \%)$ & $64(79.0 \%)$ & & 15 (22.7\%) & $51(77.3 \%)$ & \\
\hline Aberrant & $41(21.5 \%)$ & 150 (78.5\%) & & 32 (18.8\%) & 138 (81.2\%) & \\
\hline E-cadherin & & & 0.737 & & & 0.020 \\
\hline Normal & $50(21.0 \%)$ & $188(79.0 \%)$ & & 36 (17.6\%) & 169 (82.4\%) & \\
\hline Aberrant & $8(23.5 \%)$ & 26 (76.5\%) & & $11(35.5 \%)$ & 20 (64.5\%) & \\
\hline AFP & & & 0.643 & & & 1 \\
\hline - & $56(21.2 \%)$ & $208(78.8 \%)$ & & 45 (19.7\%) & 183 (80.3\%) & \\
\hline+ & $2(40.0 \%)$ & $3(60.0 \%)$ & & $1(20.0 \%)$ & $4(80.0 \%)$ & \\
\hline unknown & $0(0 \%)$ & 3 (100\%) & & $1(33.3 \%)$ & $2(66.7 \%)$ & \\
\hline VEGFR2 & & & 0.899 & & & 0.647 \\
\hline - & $6(18.2 \%)$ & 27 (81.8\%) & & $6(20.0 \%)$ & $24(80.0 \%)$ & \\
\hline+ & $25(20.7 \%)$ & $96(79.3 \%)$ & & 23 (21.5\%) & 84 (78.5\%) & \\
\hline++ & $20(22.0 \%)$ & 71 (78.0\%) & & 12 (15.8\%) & 64 (84.2\%) & \\
\hline
\end{tabular}


ARID1A AT-rich interactive domain 1A, BMI body mass index, MSI microsatellite instability, MSI-H microsatellite instability-high, MSS microsatellite stable, $P D$ - $L 1$ programmed cell death-ligand 1, $A F P$ alpha-fetoprotein, VEGFR2 vascular endothelial growth factor receptor 2

a Pearson's chi-square test (or Fisher's exact test) was used in statistical analyses.

\section{Table 2}

Univariate and multivariate COX analysis of overall survival

\begin{tabular}{|c|c|c|c|c|c|c|c|c|}
\hline \multirow{2}{*}{ Valuables } & & \multicolumn{4}{|c|}{ Univariate Cox analysis } & \multicolumn{3}{|c|}{ Multivariate Cox analysis } \\
\hline & & $P$ & $\mathrm{HR}(95 \% \mathrm{CI})$ & & Hazard Ratio & $\mathrm{P}$ & $\mathrm{HR}(95 \% \mathrm{CI})$ & Hazard Ratio \\
\hline \multirow[t]{2}{*}{ ARID1A protein } & negative & - & 1 & & & & & \\
\hline & positive & 0.002 & $0.416(0.241-0.718)$ & $\operatorname{Ber}$ & & 0.023 & $0.523(0.299-0.914)$ & \\
\hline \multirow[t]{2}{*}{ Gender } & male & - & 1 & & & & & \\
\hline & female & 0.601 & $1.167(0.655-2.081)$ & $\mapsto$ & $\longrightarrow$ & & & \\
\hline \multirow[t]{2}{*}{ Age } & $\leqslant 65 y$ & - & 1 & & & & & \\
\hline & $>65 y$ & 0.003 & $2.245(1.316-3.828)$ & & $\longmapsto$ & 0.004 & $2.200(1.278-3.787)$ & $\longrightarrow$ \\
\hline \multirow[t]{2}{*}{ T stage } & $\mathrm{T} 2 \sim \mathrm{T} 3$ & - & 1 & & & & & \\
\hline & $\mathrm{T} 4$ & 0.002 & $2.214(1.332-3.771)$ & & $\longmapsto \longrightarrow$ & 0.009 & $2.031(1.198-3.443)$ & $\longmapsto$ \\
\hline \multirow[t]{2}{*}{ N stage } & $\mathrm{N} 0 \sim \mathrm{N} 2$ & - & 1 & & & & & \\
\hline & N3 & 0.003 & $2.524(1.380-4.617)$ & & $\longmapsto$ & 0.009 & $2.282(1.233-4.225)$ & $\longmapsto$ \\
\hline \multirow[t]{2}{*}{ Tumor size } & $<3.5 \mathrm{~cm}$ & - & 1 & & & & & \\
\hline & $\geqslant 3.5 \mathrm{~cm}$ & 0.058 & $1.665(0.983,2.822)$ & & $\longrightarrow$ & & & \\
\hline
\end{tabular}

\section{Figures}


a

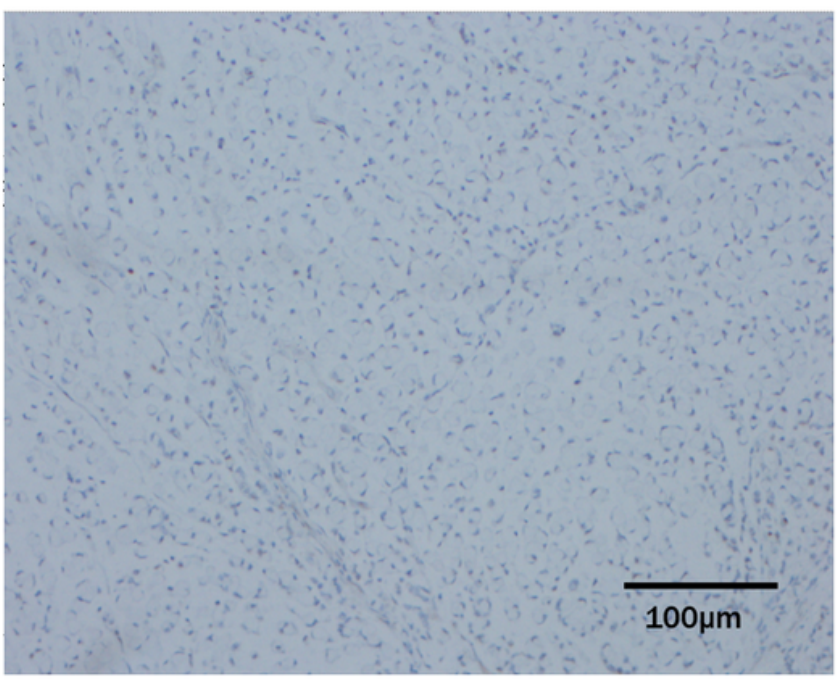

c

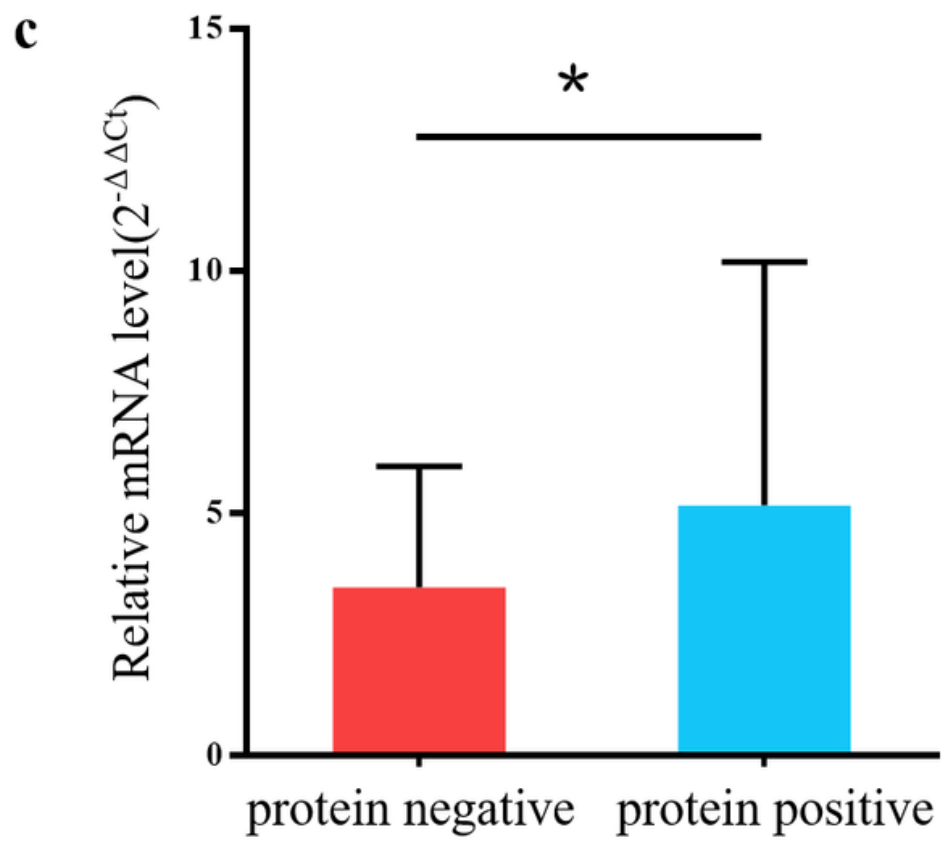

b

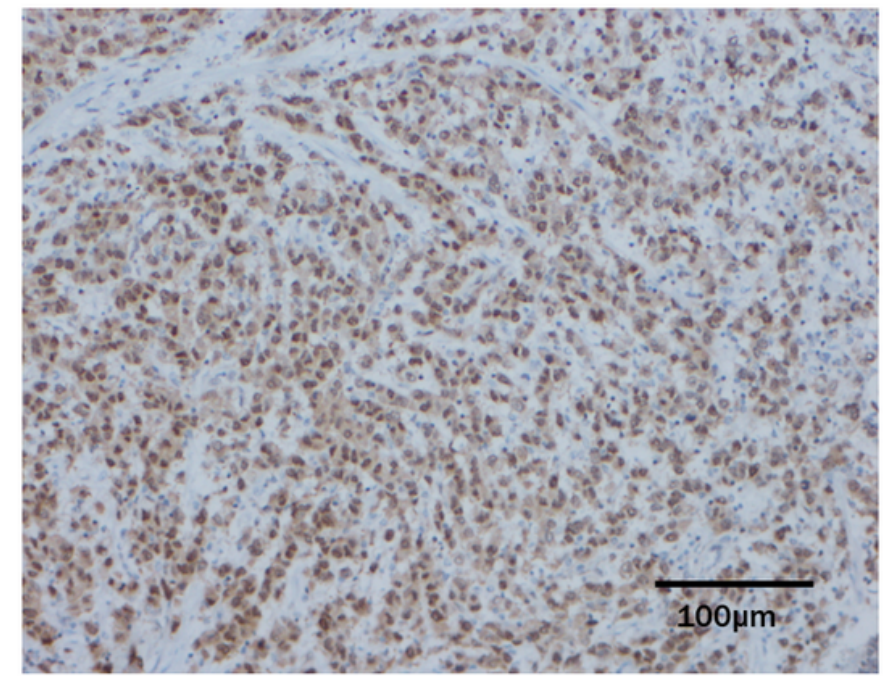

protein positive

\section{Figure 1}

Representative immunohistochemical staining images of ARID1A in GC (a) Negative ARID1A staining in tumor tissues. (b) Positive ARID1A staining in tumor tissues. Scale bars $=100 \mu \mathrm{m}$. (c) Relative mRNA level of ARID1A in protein negative and positive group. 
$\mathbf{a}$

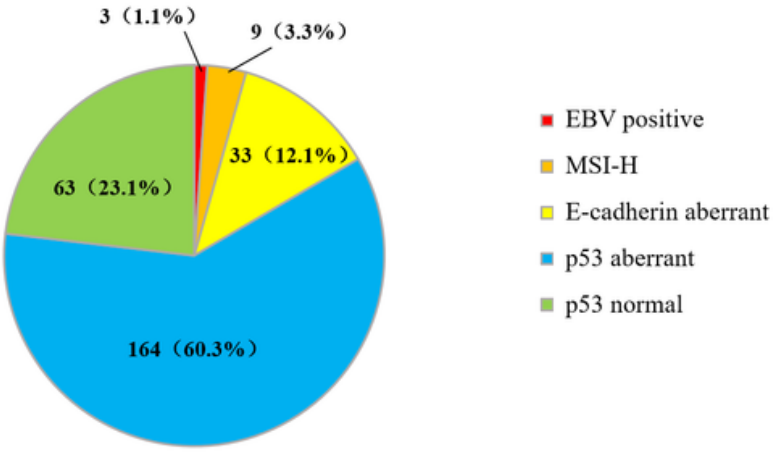

b

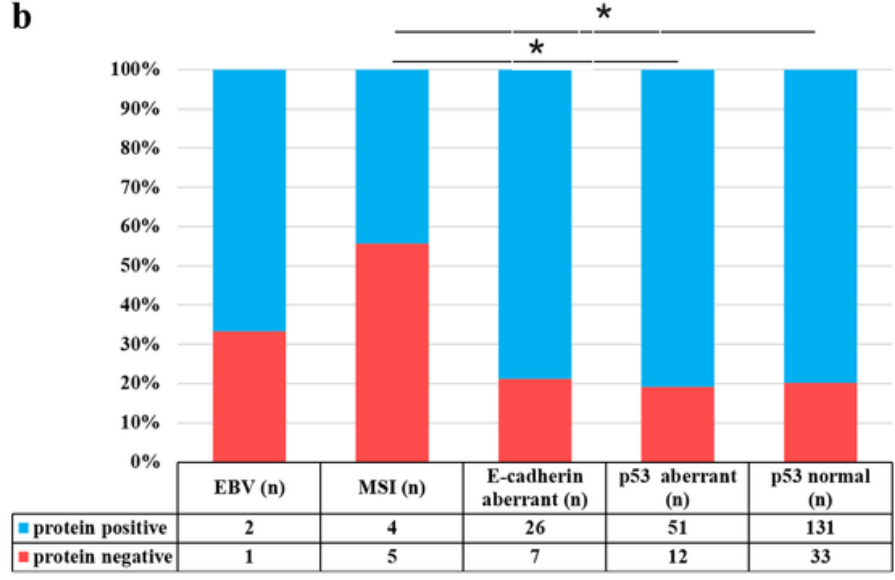

Figure 2

(a) Pie charts of five molecular subtypes of GC (b) The protein expression level of ARID1A in five molecular subtypes of GC. 

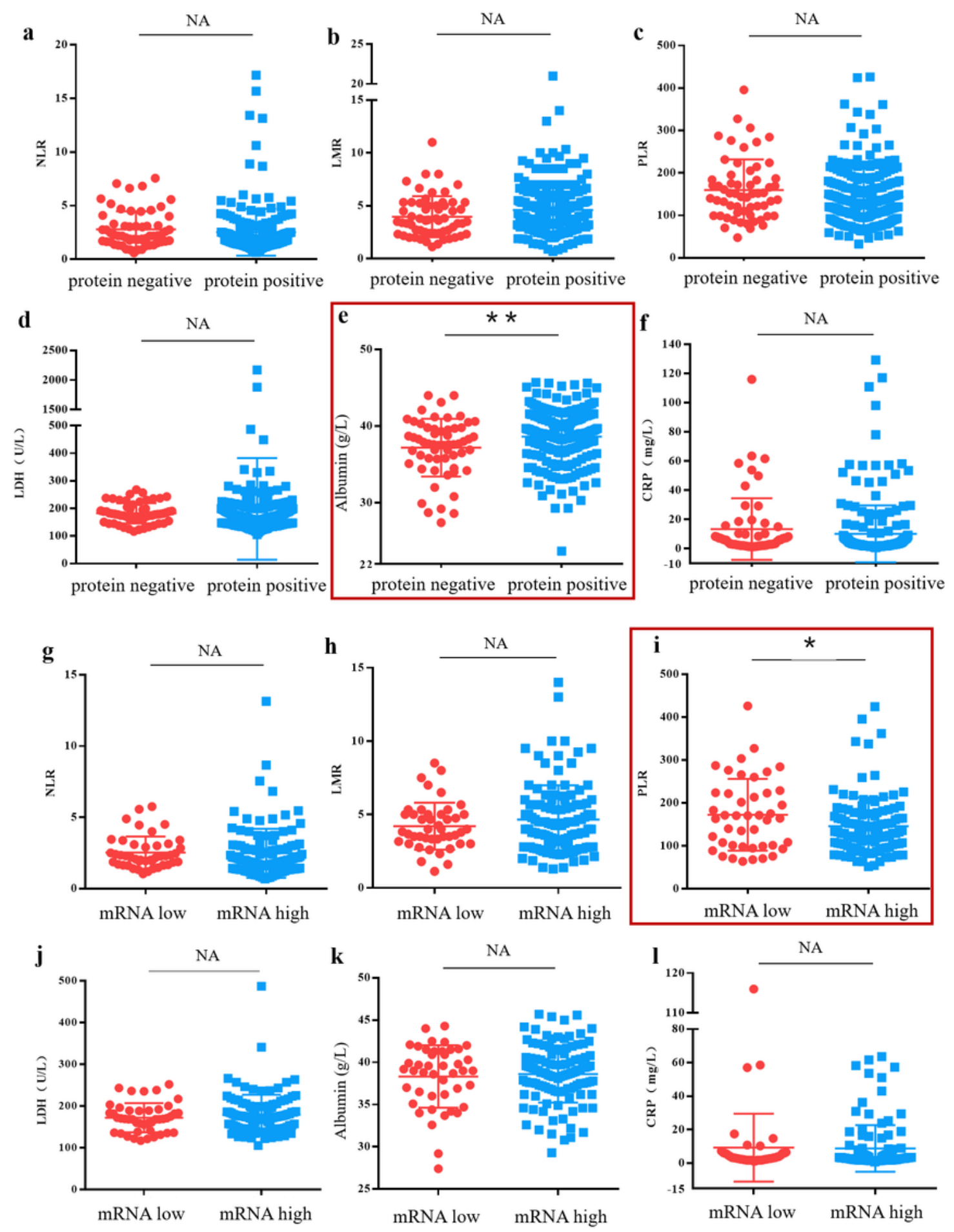

\section{Figure 3}

Relationship between ARID1A expression pattern and systemic inflammatory markers (a-f) Relationship between ARID1A protein expression and systemic inflammatory markers. (g-l) Relationship between ARID1A mRNA expression and systemic inflammatory marker. 

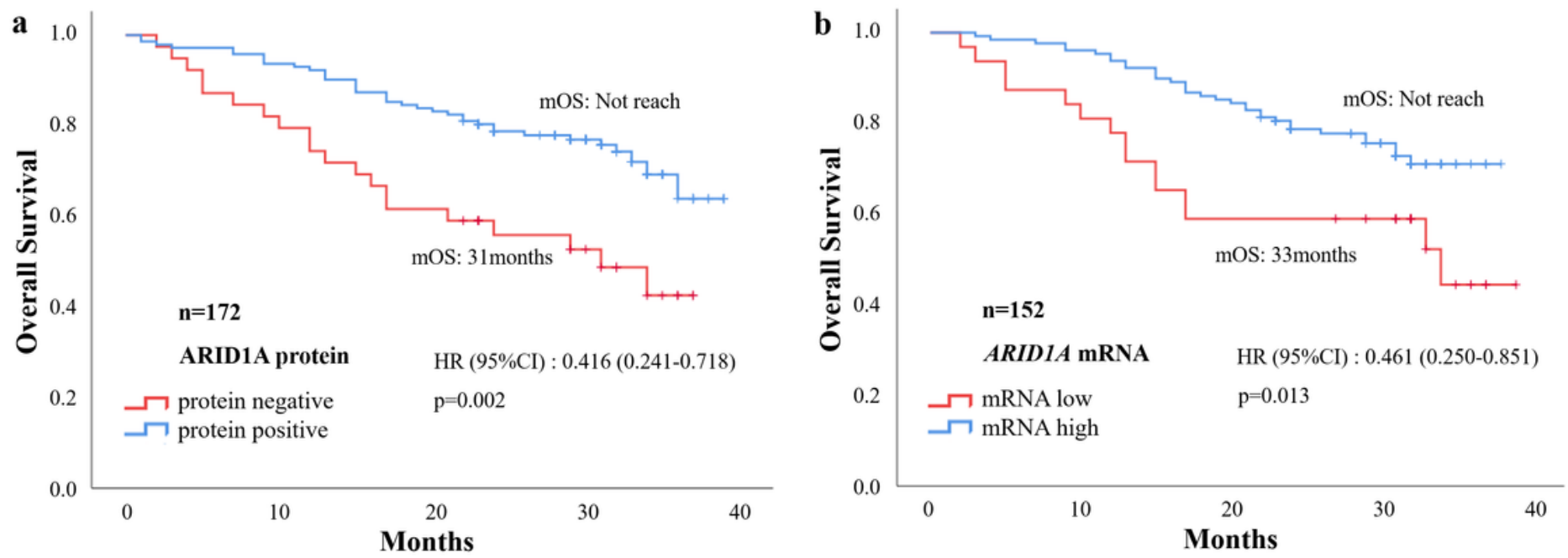

\section{Figure 4}

Kaplan-Meier survival curves for patients with GC in the (a) ARID1A protein group and the (b) ARID1A mRNA group. 


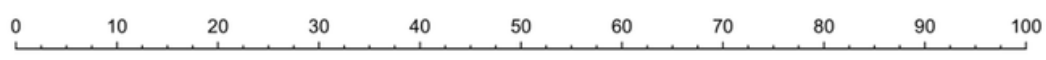
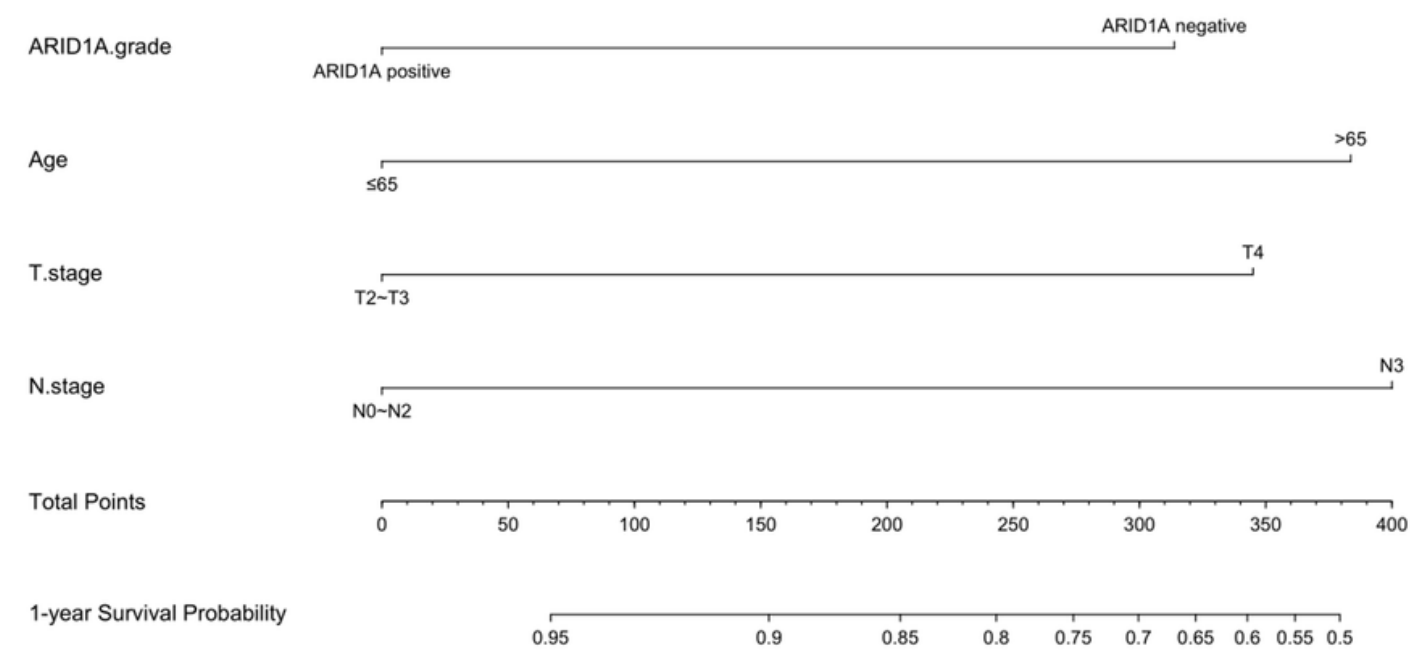

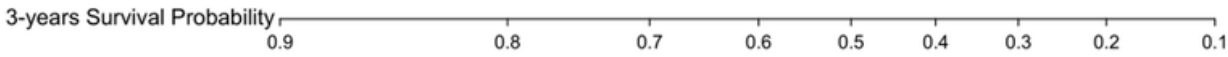
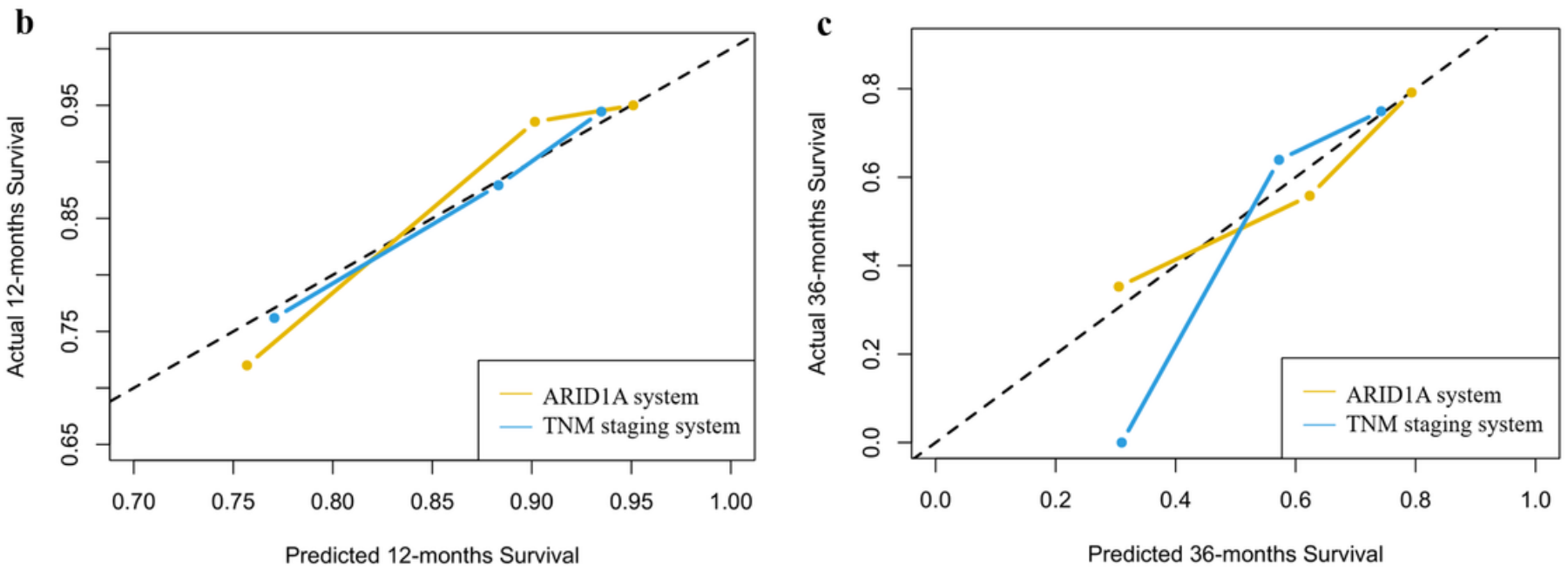

Figure 5

Development and validation of the prognostic nomogram (a) Prognostic nomogram predicting 1-and 3year survival probability for patients with GC. (b-c) Calibration curves of our nomogram and the 8th edition of the AJCC staging system for (b)1- and (c)3-years OS.

\section{Supplementary Files}

This is a list of supplementary files associated with this preprint. Click to download.

- TabS1.docx 